\title{
Activation Detection in fMRI Data via Multi-Scale Singularity Detection
}

\author{
Ralf Mekle $^{a}$, Andrew F. Laine ${ }^{a, b}$, Gerard M. Perera ${ }^{b}$, Robert DeLaPaz ${ }^{b}$ \\ ${ }^{a}$ Department of Biomedical Engineering, Columbia University, \\ ${ }^{b}$ Department of Radiology, Columbia-Presbyterian Medical Center
}

\begin{abstract}
Detection of active areas in the brain by functional magnetic resonance imaging (fMRI) is a challenging problem in medical imaging. Moreover, determining the onset and end of activation signals at specific locations in 3-space can determine networks of temporal relationships required for brain mapping. We introduce a method for activation detection in fMRI data via wavelet analysis of singular features. We pose the problem of determining activated areas as singularity detection in the temporal domain. Overcomplete wavelet expansions at integer scales are used to trace wavelet modulus maxima across scales to construct maxima lines. From these maxima lines, singularities in the signal are localized corresponding to the onset and end of an activation signal. We present results for simulated phantom waveforms and clinical fMRI data from human finger tapping experiments. Different levels of noise were added to two waveforms of phantom data. No assumptions about specific frequency and amplitude of an activation signal were made prior to analysis. Detection was reliable for modest levels of random noise, but less precise at higher levels. For clinical fMRI data, activation maps were comparable to those of existing standard techniques.
\end{abstract}

Keywords: Singularity detection, fMRI, brain imaging, wavelet modulus maxima.

\section{INTRODUCTION}

Functional magnetic resonance imaging (fMRI) is a noninvasive method of measuring spatially distributed brain activity as a function of local vasodilations ${ }^{1}$, which are characterized by hemodynamic responses. FMRI techniques are based on changes associated with the ratio of oxyhemoglobin to deoxyhemoglobin commonly referred to as the BOLD effect, i.e., Blood Oxygenation Level Dependent. This implies that the level of blood oxygenation acts as an inherent contrast agent giving rise to temporal signal changes of $\mathrm{T}_{2}{ }^{*}$ approximately $2 \sim 6 \%$ from a baseline (resting state) compared to an activation state. Thus, an MR pulse sequence that is sensitive to a magnetic susceptibility variation can be used to observe these changes in signal as a function of time ${ }^{1,2}$. Typically, an Echo Planar Gradient Echo Sequence (EPI) is used for image acquisition.

The ultimate goal of our method of analysis of 4-D fMRI data ( 3 spatial dimensions and time) is to be able to identify BOLD activations including an initial dip of an activation. An initial dip prior to activation ("negative BOLD response", see Figure 1) has been consistently observed only in high-field systems and is still under debate ${ }^{3}$. 4 . For analysis two anatomic representations are of interest, (1) identification of small structures that form the functional anatomic units of the brain, such as the cortical columns (0.5-1 mm diameter) in the visual cortex ("functional localization"), and (2) the macro structural relationships between activated regions, both spatially and temporally, which can describe network relationships between brain regions ("connectivity").

Due to small magnitude of detectable signals, scanner-induced noise, and intrinsic biological heterogeneity, fMR images typically have poor signal-to-noise-ratio (SNR). Problems with BOLD methods have been attributed to the presence of artifacts associated with head and/or vessel motion ${ }^{5}$, as well as vascular inflow ${ }^{6.7}$, and drainage effects ${ }^{8}$. Hence, detection and localization of areas of activation is a difficult task challenging state-of-the-art methods of acquisition.

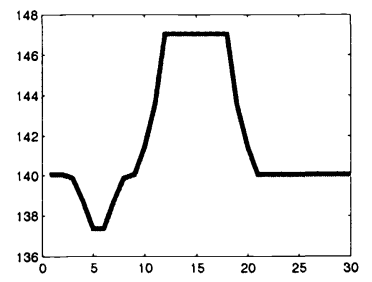

Figure 1: Schematic waveform of an activation signal including an initial dip ("negative BOLD response").

Most common fMRI experiments are block designs with "On" (activation) and "Off" (baseline) blocks of certain duration for specific activation tasks (see, e.g. ${ }^{9}, 10$ ). In addition to block design experiments, single event fMRI has been used more

Wavelet Applications in Signal and Image Processing VIII, Akram Aldroubi, Andrew F. Laine,

Michael A. Unser, Editors, Proceedings of SPIE Vol. 4119 (2000) @ 2000 SPIE · 0277-786X/00/\$15.00 
recently ${ }^{11,12}$. Several analysis methods of fMRI data already exist, including cross-correlation ${ }^{13,9}$, sinusoidal regression ${ }^{10}$, and general linear model ${ }^{14}$. More recently, fMR images have been analyzed using mutual information ${ }^{15}$, contextual clustering $^{16}$, sulcal basins ${ }^{17}$, fuzzy logic clustering ${ }^{18}$, neural networks ${ }^{19}$, and Bayesian inference ${ }^{20}$.

In comparison, our approach is to perform activation detection in fMRI data via multi-scale wavelet analysis in the temporal domain. Wavelet based denoising schemes have been applied prior to the detection of activation 21,22 . Activation detection using an orthogonal wavelet decomposition with a two-step statistical testing procedure has also been reported ${ }^{23}$. Our method poses the problem of determining areas of activation as singularity detection. Sharp variations of the signal corresponding to the onset or end of activations are traced in scale-space via maxima lines of wavelet modulus ${ }^{24}$. Three parameters ("length", "strength", and "Lipschitz alpha") are computed for each maxima line and thresholded. In addition, interscale criteria are applied to the thresholded maxima lines to separate singularities corresponding to activation from those of noise. Only singularities that meet all conditions are "accepted" as true signal transitions, or activations.

We suggest that multi-scale methods could prove superior to single-scale spatial domain testing, since the analysis functions can be locally adapted to signal properties, e.g. two different voxels/pixels having different activation patterns. Also, wavelets can better analyze non-stationary events of hemodynamics, since they are the time-frequency method of choice.

\section{METHODS}

\subsection{Overview}

In principle, our method of analysis includes preprocessing steps of background elimination, oversampling of the acquisition signals, and the localization of activated areas through multi-scale singularity detection. We validated our detection procedure by applying it to simulated phantom and human fMRI data obtained from finger tapping experiments on volunteer subjects.

\subsection{Preprocessing: Elimination of Background Pixels}

Since our analysis is restricted to intra-cranial pixels, background pixels are eliminated through a simple thresholding process. Each pixel of a slice, for which the maximum intensity of its time series is below a certain threshold is set to zero, i.e. its entire time series is set to zero. These pixels are excluded from further processing. The threshold is computed as a fraction of the maximum intensity value of the 3-D dataset (2-D slices in time). The fraction is chosen empirically for each data set as in ${ }^{19}$. For our data acquired on a 1.5 Tesla scanner it was set to the value 0.1 .

\subsection{Preprocessing: Oversampling of Acquisition Signals}

We oversample the acquisition signals by rates of 4 to 8 times the original sampling using cubic or cubic spline interpolation. Filtering oversampled signals results in smoother representations in the transform domain by allowing us to apply longer discrete filters (more taps). The oversampling procedure is completely invertible, shift-invariant, and preserves activations at the original sampling locations.

\subsection{Overcomplete Wavelet Expansions at Integer Scales}

The wavelet transform of a function $f$ is defined as

$$
W f(u, s)=\int_{-\infty}^{+\infty} f(t) \frac{1}{\sqrt{s}} \psi^{*}\left(\frac{t-u}{s}\right) d t
$$

For the continuous wavelet transform the signal is projected on a family of translated and dilated basis functions (wavelets)

$$
\psi_{u, s}(t)=\frac{1}{\sqrt{s}} \psi\left(\frac{t-u}{s}\right)
$$

Both, translation and dilation parameters are continuous, and the transform corresponds to the correlation of the function $f$ with a particular wavelet $\psi_{u, s}(t)$. The amplitude of the wavelet transform therefore tends to be maximum at those scales and locations where the signal most resembles the analysis template (waveform), thus similar to "matched filter" approaches. Overcomplete discrete representations, including the dyadic wavelet transform ${ }^{25}$ compute the continuous wavelet transform at integer values of the translation parameter $u$, but only at dyadic scales (scale parameter $s$ is always some power of 2). However, if the activation signal in the temporal domain is not best characterized at a dyadic scale, activations might go undetected, since they will only yield small coefficient values. Thus, we extend overcomplete expansions to include more scales by computing integer scales in a discrete implementation. This enabled us to trace singularities produced by activation signals more precisely in the scale-space domain. A fast algorithm implementation of the wavelet transform at integer scales is described in ${ }^{26}$. Mirror extension was used to treat boundary effects, and the first derivative of a quartic B-spline enlarged 
by a factor of 2 was selected as a basis function. This wavelet is truly symmetric around zero and has more discrete filter taps than the corresponding wavelet without enlargement centered around $1 / 2$. According to the rule of differentiation for B-splines the resulting wavelet is a cubic B-spline 26 . The quartic B-spline is depicted in Figure 2(a), and the corresponding first derivative wavelet is shown in Figure 2(b). A first derivative wavelet was intentionally chosen, since it has been shown to be suitable for purposes of detection 24.27 . Moreover, these wavelets were selected, because they are compactly supported and approximate derivatives of Gaussian functions ${ }^{28}$. Aside from optimal time-frequency properties, Gaussian functions generate a causal scale space (in a sense that a coarse scale depends exclusively on the previous finer scale) ${ }^{29}$. The last property makes possible scale-space tracking of emergent features.

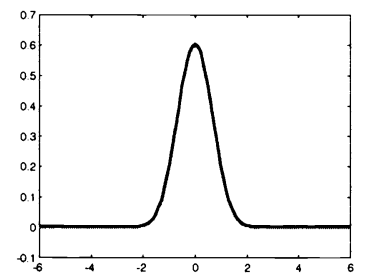

(a)

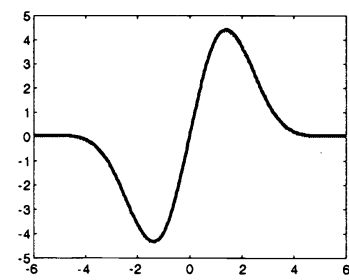

(b)

Figure 2: (a) Quartic B-spline enlarged by a factor 2. (b) First derivative cubic B-spline analysis wavelet.

To demonstrate an expansion over integer scales Figure 3 shows a simulated activation signal of a "boxcar" together with its wavelet transform computed at integer scales 1 to 10. In Figure 3(a) a noiseless signal is transformed, and in Figure 3(b) and Figure 3(c) Gaussian white noise of $1 \%$ and 3\% of baseline intensity, respectively, was added to the signal. In all cases the signals were oversampled by a factor of 4 prior to analysis. Note the strong changes of the signal, reflected as large negative (white) or large positive (black) wavelet coefficients across integer scales. The characteristic of the expansion indicates rising and falling edges of the signal correctly for all three cases. Note also that no assumptions about a specific frequency (width) and amplitude of an activation signal need to be made prior to analysis. This is a clear advantage over methods such as the cross-correlation analysis. From Figures 3(b) and 3(c) it can be seen that with increasing noise a distinction between wavelet maxima due to signal and those due to noise is more difficult at finer scales, but that only maxima due to true signal are preserved into the coarser scales. Figure 3(d) depicts scales 2 and 9 from the three cases to support this claim.

\subsection{Localization of Activations through Singularity Detection}

Our method to localize activation through singularity detection is based on the observation that for each location $(x, y, z)$ the time course of a voxel/pixel can be considered as a piecewise regular function. Thus, a wavelet transform along the temporal dimension yields a sparse representation of the data with most coefficients close to zero (vanishing property of the wavelet transform ${ }^{30}$ ). A few large coefficients are obtained at time points, where the signal consists of jump-like changes or singularities from a baseline to an activation state or vice versa. Singularity detection can be undertaken by describing the local regularity of a signal. In our approach we took advantage of the ability of the wavelet transform to characterize the local regularity of functions. The mathematical background justifying this method is described in 24.31 . For a given signal we compute all modulus maxima of its wavelet transform and chain maxima across scales to obtain maxima lines. If the signal is noisy, maxima lines due to noise are mostly concentrated at fine scales, whereas maxima lines due to signal changes should be persistent across coarser scales. Mathematically, singularity detection can be carried out by finding the abscissa where the wavelet modulus maxima converge at finer scales. If no wavelet modulus maxima exist at fine scales for a point $t=u$, it was shown that the signal is regular in $u$ (Lipschitz $n$, where $\left.\psi(t) \in \mathbb{C}^{n}\right)^{24}$.

The major task is to distinguish singularities caused by noise fluctuations from those that are generated from sharp signal transitions. Similar to the approach of Evertsz et al..$^{32}$ three criteria are used: "Length, strength, and Lipschitz alpha". Only maxima lines that persist across all scales of analysis are considered as true signal transitions, since noise fluctuations should have less persistence in scale-space. In addition, the strength of a maxima line is computed as the sum of the modulus maxima along the line (across scales). This criterion reinforces the first, since longer maxima lines tend to have larger values for their strength, and strong signal variations yield wavelet coefficients with large amplitudes resulting in values of greater magnitude. These two criteria may not be sufficient, since noise fluctuations can be of similar magnitude as signal variations, and signal changes may be corrupted by noise influence. Thus, we try to characterize the regularity of singularities through their Lipschitz exponent $\alpha$. In general, the point-wise regularity of a function can be characterized by Lipschitz exponents. Mallat and Hwang 24 showed that Lipschitz exponents could be estimated from the decay of wavelet modulus maxima. 

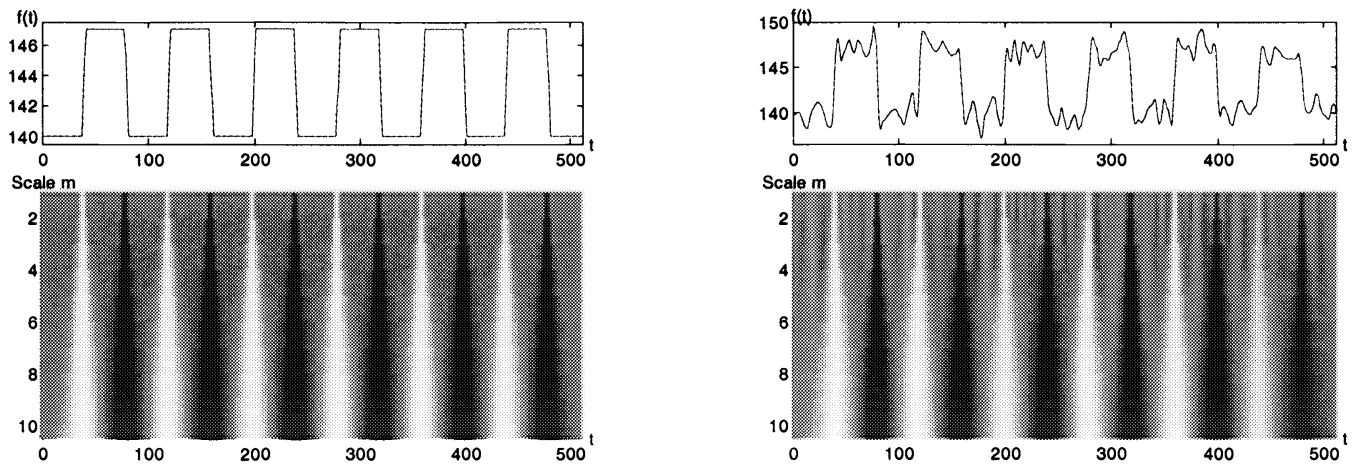

(a)

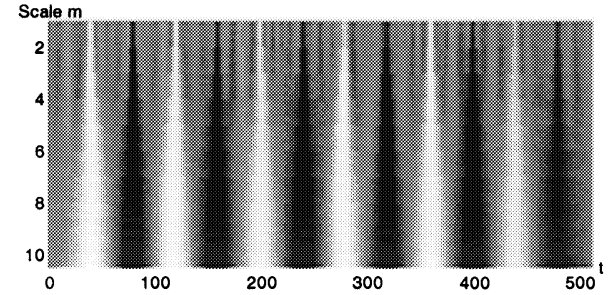

(b)

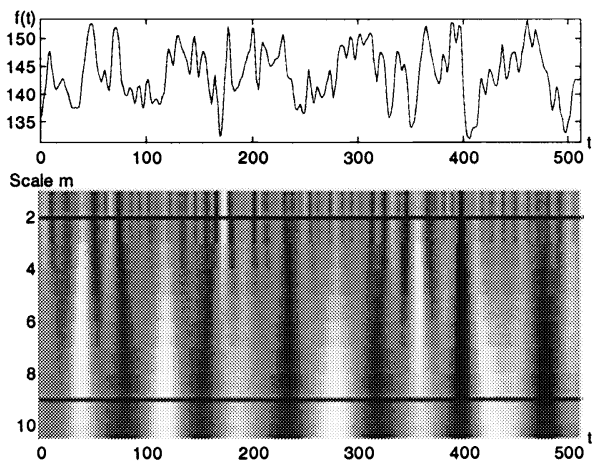

(c)

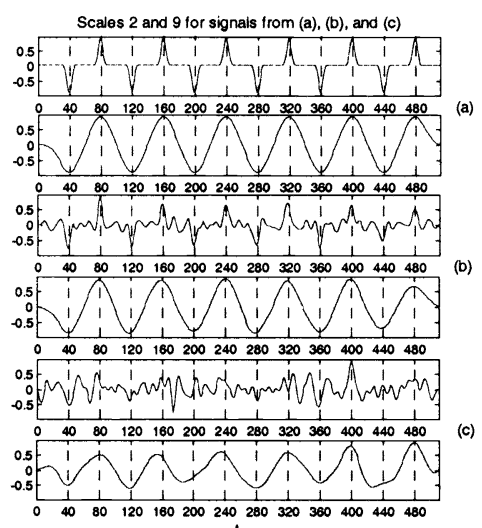

(d)

Figure 3: Expansion of phantom data at integer scales: Activation signal of a boxcar phantom and its wavelet transform at integer scales 1-10. Black, grey and white points correspond respectively to positive, zero and negative wavelet coefficients. In (a) a noiseless signal is transformed, and in (b) and (c) Gaussian white noise of $1 \%$ and $3 \%$ of baseline intensity, respectively, was added to the signal. In all cases the signals were oversampled by a factor of 4 prior to the transform. Part (d) depicts scales 2 (top) and 9 (bottom) from parts (a), (b), and (c) for comparison.

More specifically, this holds for isolated singularities, i.e. non-oscillating singularities, that "live" in the "cone of influence"

$$
|W f(u, s)| \leq A s^{\alpha+1 / 2}
$$

where $A$ is a constant and $s$ the scale parameter. The "cone of influence" of a singularity that converges to $t=v$ is defined as $|u-v| \leq C s$, where $[-C, C]$ is the support of the mother wavelet $\psi$. Equation (3) is equivalent to

$$
\log _{2}|W f(u, s)| \leq \log _{2} A+\left(\alpha+\frac{1}{2}\right) \log _{2} s
$$

Hence, the Lipschitz regularity can be estimated from the slope of $\log _{2}|W f(u, s)|$ as a function of $\log _{2} s$ along the maxima lines converging to $v$. Since the scale $s$ should be smaller than the distance between two consecutive singularities, we can estimate Lipschitz $\alpha$ from the decay slope of $\log _{2}|W f(u, s)|$ for the first three integer scales of our transform. The wavelet used in our analysis has one vanishing moment, and therefore only Lipschitz exponents smaller or equal to 1 can be measured, which is sufficient for our purposes. Essentially, singularities with more negative exponents should be attributed to noise, whereas signal transitions should have more positive exponents, since noise is highly singular (negative $\alpha$ ). Note that a step edge is Lipschitz 0 , and activation signals following a boxcar stimulation paradigm will have singularities close to step edges (in the ideal noise-free case). Threshold values for all parameters were obtained empirically for each data set. Initial settings were obtained from sample data points, and during processing all quantities for strength and Lipschitz alpha of maxima lines that satisfied the length criterion were recorded. Threshold values were then adjusted to the characteristics of each data set.

Thus, we identified singularities as onset and end of activations that passed these criteria. The sign of the wavelet transform can also provide information about whether a rising or a falling edge has been detected. An example for this procedure for a time-course of clinical fMRI data is shown in Figure 4. The oversampled signal from clinical fMRI data is shown in Figure 
4(a) (solid) displayed together with a schematic of an idealized (noise-free) signal (dashed). Wavelet modulus maxima are shown in Figure 4(b). Figure 4(c) displays the maxima lines for scales 1-10 before thresholding, and Figure 4(d) shows the maxima lines after thresholding. As mentioned previously, the expansion was carried out with a first derivative cubic spline wavelet. The resulting activation map with respect to the temporal domain assigns " 1 " to activated time points and " 0 " otherwise (see Figure 4(e)). Detected singularities are indicated by bold lines shown in Figure 4(e).

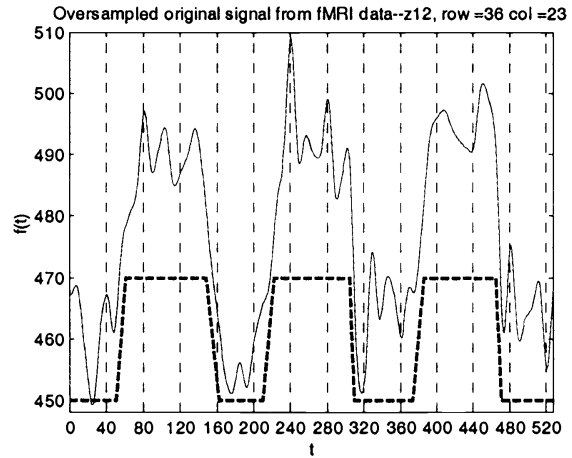

(a)

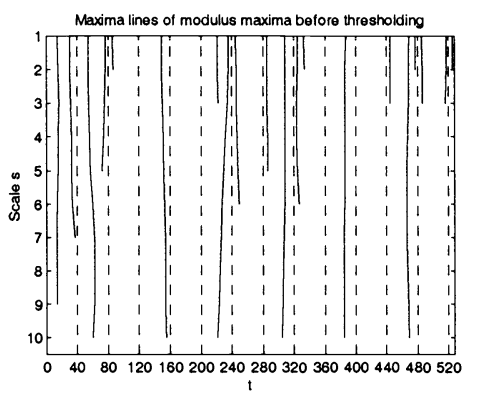

(c)

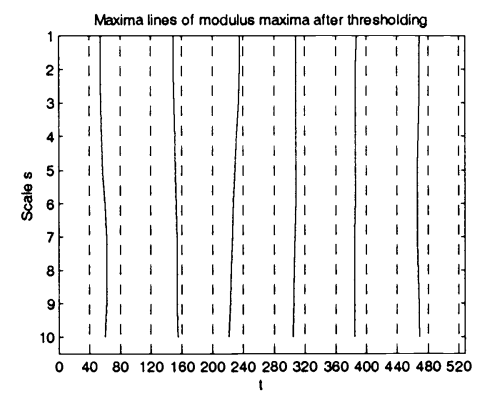

(d)

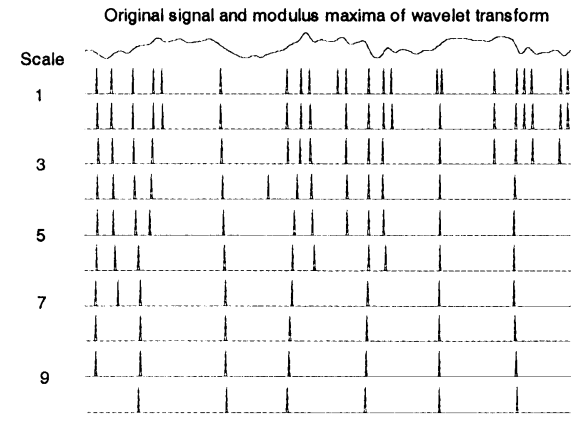

(b)

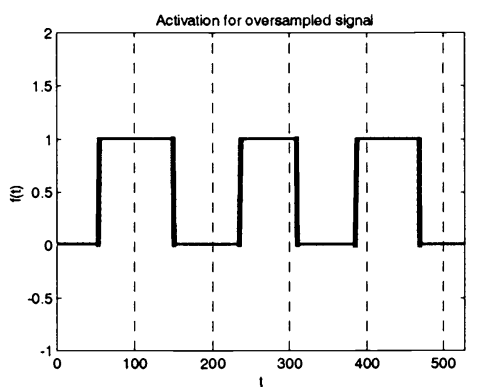

(e)

Figure 4: Analysis of clinical fMRI data: An example of determining activation through singularity detection by tracing wavelet transform modulus maxima across scales: The oversampled signal from clinical fMRI data is shown in (a) (solid) together with a schematic of an idealized (noise-free) signal (dashed). Wavelet modulus maxima are shown in part (b). Part (c) displays the maxima lines for scales 1-10 before thresholding, and part (d) the maxima lines after thresholding. The expansion was carried out with a first derivative cubic spline wavelet. Activation for the oversampled signal with respect to the temporal domain is shown in (e), where the detected singularities are indicated by bold lines.

For expository purposes Figures 3 and 4 show examples for the first 10 integer scales of analysis. However, we typically carried out our analysis for 25 scales. When computing even coarser scales $(>25)$, undesirable boundary effects start to affect detection of singularities.

Since initial results using these three criteria did not seem to provide a means for reliably distinguishing singularities, we added additional criteria to better characterize the regularity of singularities. Instead of only considering wavelet modulus maxima, another quantity, the so-called "wavelet transform modulus sum (WTMS)", provides information about the regularity of signals. This quantity was introduced $\mathrm{in}^{33}$ and used for the denoising of 1-D signals and 2-D images. The WTMS for a point $u$ is defined as

$$
N f(u, s)=\int_{|t-u| \leq c s}|W f(t, s)| d t
$$

where the integral is taken over the cone of influence mentioned above. Using Theorem 1 from ${ }^{24}$ it can be shown that the WTMS is bounded by

$$
N f(u, s) \leq A^{\prime} s^{\alpha+1}
$$

Hsung and collaborators then derived the criteria of interscale ratios and interscale differences of the WTMS for dyadic scales $s=2^{j}$. These criteria describe how much the WTMS for a point $u$ should increase from one scale $s_{0}$ to the next coarser scale $s^{\prime}=\left(s_{0}+1\right)$, if the signal is regular in $u$. We derived such criteria for the transform at integer scales. The interscale ratio can then be expressed as 


$$
\frac{N\left(u,\left(s_{0}+1\right)\right)}{N\left(u, s_{0}\right)}=\left(1+1 / s_{0}\right)^{\alpha+1}=R
$$

and the interscale difference as

$$
N\left(u,\left(s_{0}+1\right)\right)-N\left(u, s_{0}\right)=A^{\prime} s_{0}^{\alpha+1}\left(\left(1+1 / s_{0}\right)^{\alpha+1}-1\right) \geq \gamma \geq 0
$$

Note that these criteria are derived from the inequality (6), so that their correct formulation should be in terms of the suprema of the WTMS at each scale. The interscale ratio $R$ is dependent on the scale $s_{0}$, and the factor $\gamma$ for the interscale difference was used to remove small irregular signals and generally depends on the amplitude of the signal. For $\gamma=0$, inequality (8) simply restates (7).

To finally determine activated pixels in a slice of the brain or simulated phantom data, i.e. to obtain an activation map with respect to the spatial distribution of activation, we applied all criteria to the extracted maxima lines. Only maxima lines that met all thresholding and regularity conditions were considered as signal transitions in the time series of a pixel corresponding to an onset or end of an activation signal. If the number of those maxima lines exceeded a number of "outliers", a pixel was considered as activated. For our studies the number of outliers was chosen to be 2 .

\section{MATERIALS FOR VALIDATION}

\subsection{Phantom Models}

We tested our detection paradigm on functional magnetic resonance data from a finger tapping task by a human and computer generated phantoms, which served as idealized models. The phantom data was modeled as single slices that contained Gaussian white noise. The assumption of Gaussian white noise is widely used, though not completely accurate, especially for low signal-to-noise ratios (SNRs), where a signal dependent bias also has to be taken into account for the Rician noise distribution $^{34}$ in magnitude images of magnetic resonance data. Nevertheless, in $^{35}$ it is shown that the Gaussian approximation is already valid for moderately high SNRs, e.g. $\mathrm{SNR}=3$. Each slice of the phantom (fixed $z$ ) consisted of $64 \mathrm{x}$ 64 data points in the $x$ - and $y$-dimensions and 128 values for the temporal domain. Various waveforms were designed for activation signals with three different levels of noise added, similar to block design experiments. The activation signals were shifted with respect to time to simulate delay. A matrix of the different types of simulated waveforms with possible parameters of shape are given in Table 1.

\begin{tabular}{|l|c|c|c|c|}
\hline \multicolumn{1}{|c|}{ Waveform } & $\begin{array}{c}\text { Magnitude of } \\
\text { Activation } \\
\text { (\% of baseline) }\end{array}$ & $\begin{array}{c}\text { Added Noise } \\
\text { (\% of baseline) }\end{array}$ & $\begin{array}{c}\text { Width of Activation } \\
\text { (\# of time points) }\end{array}$ & $\begin{array}{c}\text { Delays of Signals } \\
\text { (\# of time points) }\end{array}$ \\
\hline Boxcar & $5 \%$ & $1 \%, 2 \%, 3 \%$ & 10 & $0,5,8,10$ \\
\hline Trapezoid & $5 \%$ & $1 \%, 2 \%, 3 \%$ & 6 & $0,5,8,10$ \\
\hline
\end{tabular}

Table 1: Simulated waveforms of phantom data and experimental parameters.

\subsection{Clinical fMRI Experimental Data from 1.5 Tesla Magnet}

Data was acquired from finger tapping experiments carried out at Columbia-Presbyterian Medical Center. The corresponding stimulation task consisted of 30 seconds of finger tapping for the active state (A), followed by 30 seconds of rest for the baseline state (B). This was repeated for three cycles, i.e. (BA, BA, BA). The total time for one run was 3 minutes and 30 seconds resulting in 70 time points for each location. This task was selected because of its robustness to activate the primary motor cortex on both sides of the brain (bilateral activation). Scanning was carried out on a GE Signa MRI Scanner (1.5 T) retrofitted for Echo Planar Imaging (EPI). The scanner software version was Signa 5.7. A gradient echo sequence was used with the following parameters: $\mathrm{TE}=80 \mathrm{msec}, \mathrm{TR}=3000 \mathrm{msec}$, Flip angle $=90$, one nex, zero gap, $\mathrm{FOV}=20 * 20 \mathrm{~cm}$ in plane, matrix $64 * 64$ in plane, and slice thickness (in $z$-direction) of $7 \mathrm{~mm}$. A Bird cage quadrature head coil was used to acquire the $\mathrm{T}^{*}$ weighted images, which had voxel dimensions of $3.125,3.125$, and $7 \mathrm{~mm}$ in $x, y, z$ dimensions, respectively for the field-of-view (FOV) and matrix mentioned above. Before the analysis the slices were co-registered using Woods' algorithm ${ }^{36}$ and ratio-normalized. These standard procedures were applied to correct for artifacts due to patient movement and global signal variations that might be caused by the scanner.

\section{RESULTS}

We present results for simulated phantom data and clinical fMRI data from bilateral finger tapping experiments. In general, distinguishing singularities caused by noise and singularities generated by signal transitions proved difficult. Thresholding and regularity conditions described in Section 2.5 clearly helped to detect "true-positives", i.e. transitions corresponding to 
activation signals, and to reduce the number of "false-positives", i.e. transitions due to noise fluctuations, but could not completely reliably distinguish signal and noise. Next, we present results followed by a discussion of the influence of the parameters of maxima lines and possible improvements of our method before the concluding section.

\subsection{Phantom Models}

For phantom data the analysis was carried out with an oversampling factor of 4 , and a wavelet expansion using the first derivative cubic B-spline wavelet described in Section 2.4 was computed at 25 integer scales. As expected, correct singularity detection was strongly dependent on the level of noise added to the signal. For phantom data of the boxcar type (see Figure 5 for examples of this type of data) that resembled the time-course of stimulation paradigms with "on" and "off' blocks, perfect detection was achieved for data with $1 \%$ noise ( $1 \%$ of baseline intensity as standard deviation of added Gaussian white noise). By perfect detection we mean here not only zero "false-negatives" (missed activations) in the resulting spatial activation map, but also the correct identification of all "activation edges", i.e. the onset and end points of activation signals with respect to their time-course, as shown in Figure 5. Perfect detection could be accomplished, since a clear separation of maxima lines corresponding to signal as compared to noise was possible by thresholding. All small noise fluctuations were successfully rejected. If the level of noise was increased to $2 \%$, nearly perfect detection was still observed. A further increase to a higher level (3\% of baseline intensity) to produce singularities that were equal or even larger than those generated by activation, yielded a decreased detection performance, since maxima lines due to noise persisted across all scales. The exact time course of the onset and end of activation could not always be identified in this case. Thus, a trade-off between correctly detecting signal transitions and rejecting "false-positives" was made. Parameter values of maxima lines of strong noise fluctuations were in a similar range as those of signal variations. Applying the interscale criteria discussed in Section 2.5 across many scales led to improved rejection of "false-positives"(specificity), but at the same time to a decrease in the detection of activation edges (sensitivity). Similar behavior was observed for phantom data of the trapezoid type, where the activation signals were modeled with rising and falling edges of finite slope compared to the step edge behavior of boxcar type data at noise levels of $1 \%$ and $2 \%$. At $3 \%$ noise detection performance was less than observed in the case of boxcar type data. However, this was to be expected, since the first derivative wavelet yields higher coefficients for more rapid signal transitions, which improves detection performance.

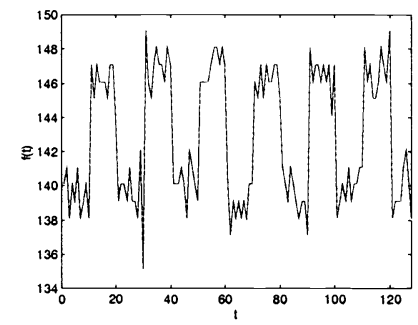

(a)

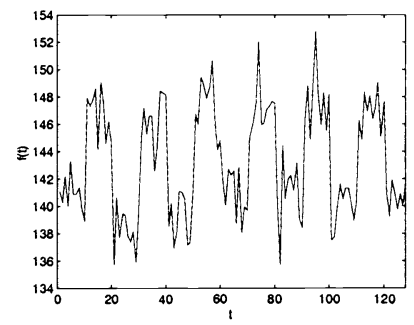

(b)

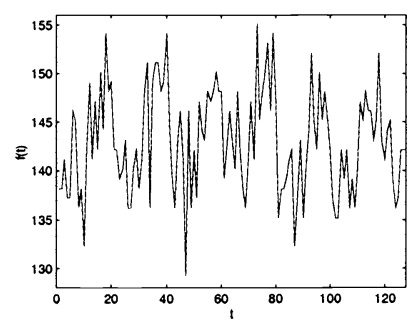

(c)

Figure 5: Examples of phantom data of boxcar type: (a) $1 \%$ noise, (b) $2 \%$ noise, and (c) $3 \%$ added noise (\% of baseline intensity).

Examples for these types of observations are given in Table 2, where we listed the number of "false-positives"(FP) for the case of zero "false-negatives" in the resulting spatial activation map. Due to the simplicity of our phantom data zero "falsenegatives" could always be achieved, even though not all onset and end points of activation signals could be detected. That the detection performance decreased with increasing noise is demonstrated by listing the percentage of correctly detected activation edges (\% DAE) shown in Table 2 .

\begin{tabular}{|l|c|c|c|c|c|c|}
\hline Waveform & \multicolumn{2}{|c|}{$1 \%$ Noise } & \multicolumn{2}{c|}{$2 \%$ Noise } & \multicolumn{2}{c|}{$3 \%$ Noise } \\
\hline & FP & \% DAE & FP & \% DAE & FP & \% DAE \\
\hline Boxcar & $\mathbf{0}$ & 100 & $\mathbf{0}$ & 99.6 & 28 & 83.7 \\
\hline Trapezoid & $\mathbf{0}$ & 100 & $\mathbf{0}$ & 99.8 & 91 & 62.2 \\
\hline
\end{tabular}

Table 2: Number of "false-positives" and percentage of correctly detected activation edges (\%DAE) for phantom data. The noise level is given in $\%$ of baseline intensity of the phantom data. The number of false-negatives in the resulting activation map was zero in all cases.

\subsection{Clinical fMRI Experimental Data from 1.5 Tesla Magnet}

We also applied our method to clinical fMRI data from bilateral finger tapping experiments carried out in a 1.5 Tesla magnet. As mentioned above, we used an oversampling factor of 8 and computed the wavelet transform at 25 integer scales for time series of single slices (fixed z-coordinate). Each slice contained 64 by 64 pixels, and 66 time points of a slice were included 
in the analysis. The first 4 time points for each slice were discarded to eliminate a large signal spike that was attributed to a spin history artifact at the beginning of image acquisition. A larger oversampling factor compared to the analysis of phantom data was chosen, in order to be able to compute more scales without severe boundary effects. Smaller factors and fewer scales were tried experimentally, but detection improved by using more scales. Initially, the observations from experiments with phantom data with increased levels of noise were confirmed. For the sample case processing (Figure 4) detection of activation signals worked very well. However, it was difficult to reject "false-positives". Only after applying the interscale criteria across many scales of our analysis, better rejection was realized.
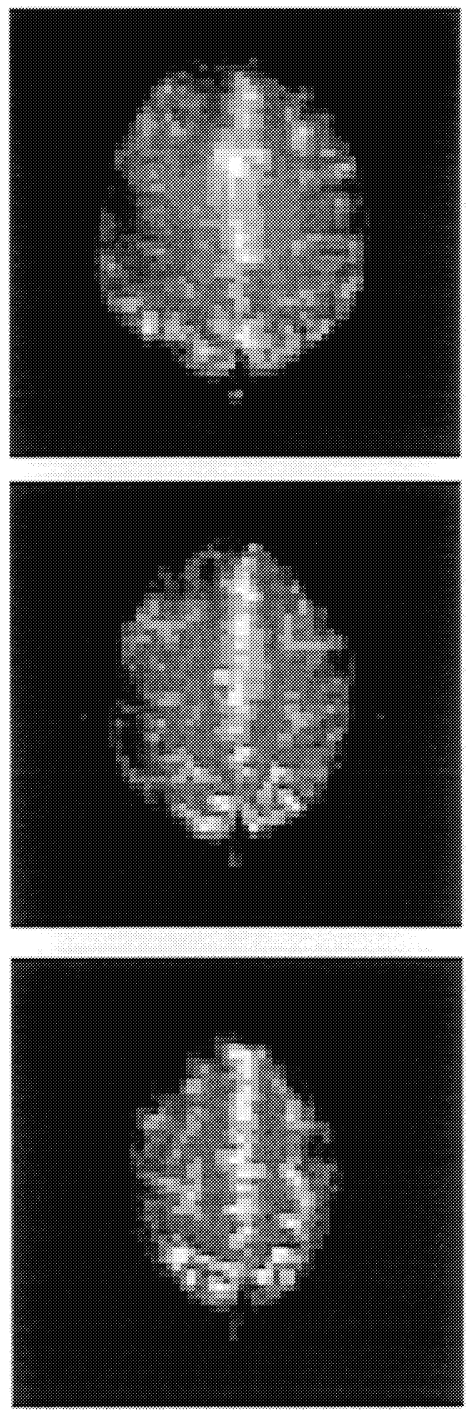

(a)
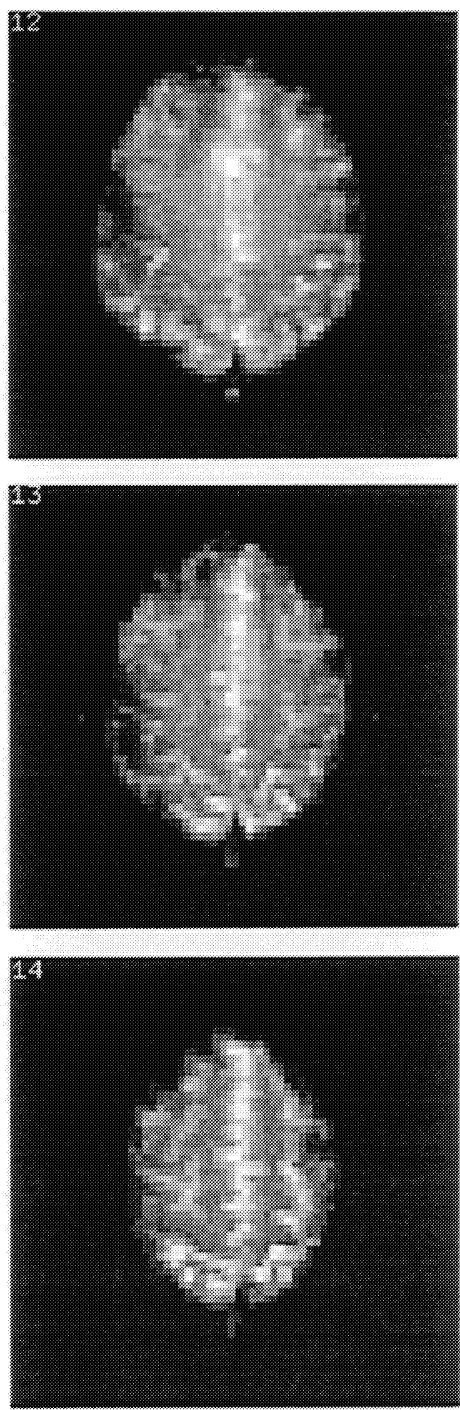

(b)
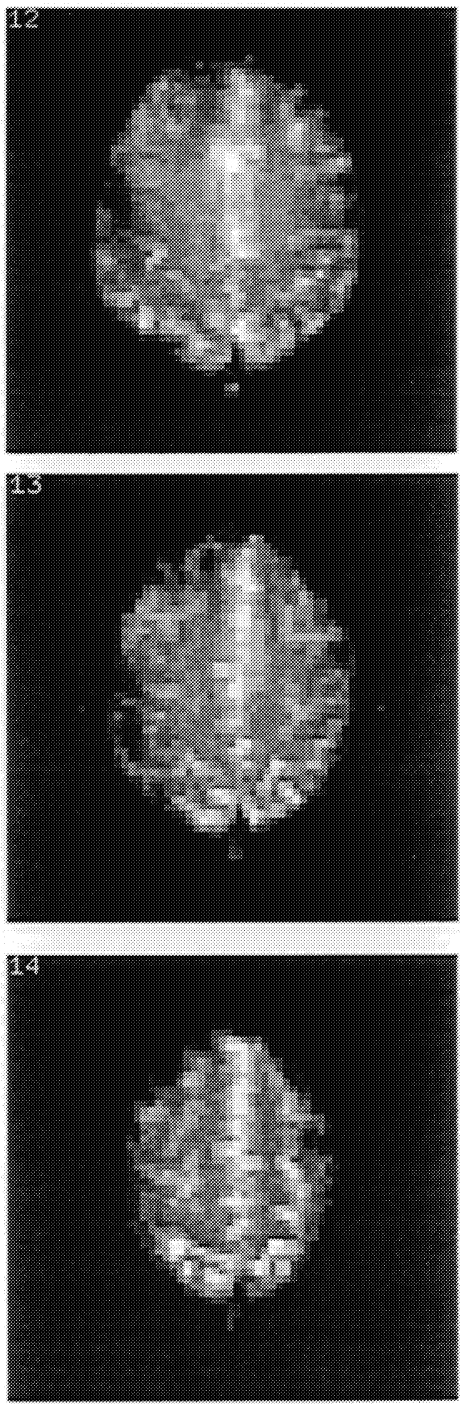

(c)

Figure 6: Color-washed activation maps for 3 slices of clinical fMRI data: Colored pixels show locations of detected activations in the brain. (a) Results from singularity detection via wavelet modulus maxima. (b) Results from cross-correlation analysis with $z$-score $\geq 3.0$. (c) Results from detection by the method of sinusoidal regression also using a $z$-score $\geq 3.0$.

Figure 6 shows the resulting activation maps for three slices of the brain containing activation obtained with three detection methods. Maps are presented as color-washed overlays on the original intensity data. In Figure 6(a) results from singularity detection via wavelet modulus maxima are shown, and Figure 6(b) presents results obtained from cross-correlation analysis with $z$-score $\geq 3$.0. Figure $6(\mathrm{c})$ shows activations detected with the method of sinusoidal regression also using a $z$-score $\geq 3.0$. The numbers in the upper left corner of the images in Figures 6(b) and 6(c) refer to the z-coordinate of the corresponding slice. Activation locations in Figure 6(a) are indicated with the same color since our method only assigns a fixed value ("l") for each activated voxel. The other two techniques map a range of $z$-scores into a color scale (the higher the $z$-score, the more yellow the pixel). 
As seen from Figure 6, results obtained with our method are very similar to those obtained with the other two standard detection techniques. The centroids of areas of activation are at the same locations, though activated regions are of smaller extent in Figure 6(a) compared to Figure 6(b) and Figure 6(c). However, note that only very few "false-positives" were observed, which for testing purposes was confirmed experimentally by viewing the time-course of activated pixels.

These results are certainly encouraging. Nevertheless, we note that in detecting activations in clinical data (similar to phantom data with noise levels of 3\%) not all onset and end points of activation signals were detected. More precisely, not all activation edges met the thresholding and regularity conditions necessary to reject "false-positives". Adjusting threshold values and regularity conditions to allow detection of more activation edges resulted in an increased number of "falsepositives". Thus, possible improvements of the method might be to develop an algorithm for adaptive parameter changes.

\subsection{Discussion}

As mentioned in Section 1 the general characteristics of fMRI data, low SNR with Rician noise that adds a signal dependent bias to the data, together with weak activation signals make correct detection of activated areas in the brain a challenging task. Our method of singularity detection via the analysis of wavelet modulus maxima was able to find small signal transitions, even, if these changes were sometimes deeply "buried" in noise. Maxima lines spanned across integer scales for all signal variations. A challenging problem remains to distinguish between singularities caused by true signal from those generated by noise or, in other words, to reject "false-positives". Unfortunately, we found that the parameters "length", "strength" and "Lipschitz alpha" take similar values for strong noise fluctuations and weak signal transitions, so that a clear separation between them was not possible. This was observed for clinical data as well as for phantom data with higher levels of noise.

To measure the local regularity of a signal through estimating Lipschitz exponents did not prove to be truly reliable. Though this measure worked well for test cases, such as step edges or discrete delta impulses, its numerical precision was limited. One explanation stems from the fact that estimating Lipschitz exponents is based on inequality (4), not on an equation. In addition, the computation of fine scales of the wavelet transform used for the estimation is limited by the finite sampling resolution of discrete data. Lipschitz exponents are estimated from the decay of wavelet modulus maxima at the finest scales, which, in theory, approach 0 , but cannot be realized in practice. Similar considerations hold for the interscale criteria, interscale ratio $R$ from (7) and interscale difference $\gamma$ from (8), which are also derived from inequalities and might not provide sufficient numerical precision to correctly characterize regular parts of a signal. Since noise fluctuations are highly singular, their Lipschitz exponents are negative. For example, a (Gaussian) white noise distribution is uniformly Lipschitz $-1 / 2-\varepsilon$, for any $\varepsilon>0^{24}$, and signal transitions have positive Lipschitz exponents, if they are not more irregular than step edges. But since noise may strongly corrupt the hemodynamic signal, Lipschitz exponents of the sum of signal and noise can be negative, if the influence of noise dominates. All interscale criteria are based on the assumptions of positive Lipschitz exponents for signal variations. Hence, rejection of "false-positives" due to noise might remove activation edges as well that do not meet the interscale criteria. This makes the correct detection of the temporal onset and end points of activation signals more difficult. One remedy could be to include information about neighboring pixels into the analysis ("clustering"16). Since our current method focused on the time-course of each voxel, it had the advantage of enabling detection of activated areas of small size. To design a mathematical model for the characteristics of Rician noise in terms of Lipschitz exponents for singularities of a signal corrupted with this type of noise is a possible direction of future work that might improve detection performance. Alternatively, the temporal pattern of detected singularities could be examined to determine true signal transitions. Certainly, this would require information about the stimulation paradigm, which would limit flexibility. Another line of improvement for our method lies in the derivation of thresholding parameters for maxima lines from the data directly. Though we record parameter values of maxima lines that span across integer scales, we have not analyzed these data in depth. The processing strategy could be simplified and sped up, if data dependent patterns for the parameters were determined.

A few additional observations are briefly summarized next. As mentioned earlier different oversampling parameters were used for phantom and clinical data. Since oversampling was carried out by standard interpolation, a larger oversampling factor resulted in more regular singularities equivalent to more positive Lipschitz exponents. Thus, for phantom data the threshold for Lipschitz alpha $T_{\alpha}$ was chosen to be slightly negative, e.g. $T_{\alpha}=-0.25$, but for the analysis of clinical data this threshold was positive, e.g. $T_{\alpha}=+0.45$. The detection performance was not influenced by different choices of the oversampling parameter. In general, changes of the threshold for Lipschitz alpha did not have a strong effect on detection performance. The method was more sensitive with respect to varying the threshold for the strength parameter. If this threshold was too low, the number of "false-positives" increased strongly. Thresholding the strength parameter with a large value and applying the interscale criteria across many scales led to better results in terms of rejection of "false-positives", while preserving true activation edges. 
For continuing validation we plan to compare our results with those from existing standards, such as cross-correlation analysis and sinusoidal regression. Further work is in progress to evaluate our detection procedure quantitatively and to increase statistical power of simulated experiments with more sophisticated phantom data. This includes adding of signal dependent Rician noise to allow our models to more closely resemble the noise characteristics of fMRI data.

\section{CONCLUSIONS}

We suggest that multi-scale singularity detection of fMRI data has the potential to precisely and adequately determine areas of activation within the brain. By detecting singularities through the tracing of modulus maxima of wavelet transform coefficients across integer scales activation signals can be identified. No assumptions about a specific frequency or time course of activation signals need to be made prior to processing. From singularity detection maps, activated brain areas can be identified, when singularities caused by noise are rejected. However, our current procedure can be improved. In particular, the characteristics of singularities created by noise can be analyzed more precisely to distinguish them from singularities produced by signal. To achieve this, the Rician noise model for fMRI data should be analyzed in the transform domain. This should reduce the number of "false positive" activations (increase specificity), while detecting more "true-positives" (increase sensitivity). Denoising through non-linear hard- or soft-thresholding 37 prior to detection, may also improve detection performance.

\section{REFERENCES}

[1] S. Ogawa, T. M. Lee, A. S. Nayak, and P. Glynn, "Oxygenation-sensitive contrast in magnetic resonance image of rodent brain at high magnetic fields," Magnetic Resonance in Medicine, vol. 14, pp. 68-78, 1990.

[2] C. T. W. Moonen, "Imaging of human brain activation with functional MRI," Biological Psychology, vol. 37, pp. 141-143, 1995.

[3] R. S. Menon, S. Ogawa, X. Hu, J. P. Strupp, P. Anderson, and K. Ugurbil, "BOLD based functional MRI at 4 Tesla includes a capillary bed contribution: echo-planar imaging correlates with previous optical imaging using intrinsic signals," Magnetic Resonance in Medicine, vol. 33, pp. 453-459, 1995.

[4] D.-S. Kim, T. Q. Duong, and S.-G. Kim, "High-resolution mapping of iso-orientation columns by fMRI," Nature Neuroscience, vol. 3, pp. 164-169, 2000.

[5] J. V. Hajnal, R. Myers, A. Oatridge, J. E. Schwieso, I. R. Young, and G. M. Byder, "Artifacts due to stimulus correlated motion in functional imaging of the brain," Magnetic Resonance in Medicine, vol. 31, pp. 283-291, 1994.

[6] J. H. Duyn, C. T. W. Moonen, R. W. d. Boer, G. H. v. Yperen, and P. R. Luyton, "Inflow versus deoxyhemoglobin effects in functional MRI using gradient echos at 1.5 T," NMR in Biomedicine, vol. 7, pp. 83-88, 1994.

[7] S. Kim, K. Hendrich, X. Hu, H. Merkle, and K. Ugurbil, "Potential pitfalls of functional MRI using conventional gradient-recalled echo techniques," NMR in Biomedicine, vol. 7, pp. 69-74, 1994.

[8] S. Lai, A. L. Hopkins, E. M. Haacke, D. A. Wasserman, P. Buckley, L. Friedman, H. Meltzer, P. Hedera, and R. Friedland, "Identification of vascular structures as a major source of signal contrast in high resolution 2-D and 3-D functional activation imaging of the motor cortex at 1.5 T," Magnetic Resonance Imaging, vol. 30, pp. 387-392, 1993.

[9] P. A. Bandettini, A. Jesmanowicz, E. C. Wong, and J. S. Hyde, "Processing strategies for time-course data sets in functional MRI of the human brain," Magnetic Resonance in Medicine, vol. 30, pp. 161-173, 1993.

[10] E. Bullmore, M. Brammer, S. C. R. Williams, S. Rabe-Hesketh, N. Janot, A. David, J. Mellers, R. Howard, and P. Sham, "Statistical methods of estimation and inference for functional MR image analysis," Magnetic Resonance in Medicine, vol. 35, pp. 261-277, 1996.

[11] O. Josephs, R. Turner, and K. Friston, "Event-related fMRI," Human Brain Mapping, vol. 5, pp. 243-248, 1997.

[12] A. M. Dale, "Optimal experimental design for event-related fMRI," Human Brain Mapping, vol. 8, pp. 109-14, 1999.

[13] P. A. Bandettini, E. C. Wong, M. S. Hinks, R. S. Tifofsky, and J. S. Hyde, "Time course EPI of human brain function during task activation," Magnetic Resonance in Medicine, vol. 25, pp. 390-397, 1992.

[14] K. J. Friston, A. P. Holmes, K. J. Worsley, J. P. Poline, C. D. Frith, and R. S. J. Frackowiak, "Statistical Parametric Maps in Functional Imaging: A General Linear Approach," Human Brain Mapping, vol. 2, pp. 189-210, 1995.

[15] A. Tsai, J. W. Fisher, C. Wible, W. M. Wells, J. Kim, and A. S. Willsky, "Analysis of Functional MRI Data Using Mutual Information," presented at Medical Imaging and Computer-Assisted Intervention - MICCAI'99, C. Taylor, A. Colchester, Eds. Cambridge, England, pp. 473-480, 1999. 
[16] E. Salli, A. Visa, H. J. Aronen, A. Korvenoja, and T. Katila, "Statistical Segmentation of fMRI Activations Using Contextual Clustering," presented at Medical Imaging and Computer-Assisted Intervention - MICCAI'99, C. Taylor, A. Colchester, Eds. Cambridge, England, pp. 481-488, 1999.

[17] G. Lohmann and D. Y. v. Cramon, "Using Sulcal Basins for Analyzing Functional Activation Patterns in the Human Brain," presented at Medical Imaging and Computer-Assisted Intervention - MICCAI'99, C. Taylor, A. Colchester, Eds. Cambridge, England, pp. 489-497, 1999.

[18] X. Golay, S. Kollias, G. Stoll, D. Meier, A. Valvanis, and P. Boesiger, "A new correlation-based fuzzy logic clustering algorithm for fMRI," Magnetic Resonance in Medicine, vol. 40, pp. 249-60, 1998.

[19] K.-H. Chuang, M.-J. Chiu, and C.-C. Lin, "Model-Free Functional MRI Analysis Using Kohonen Clustering Neural Network and Fuzzy C-Means," IEEE Transactions on Medical Imaging, vol. 18, pp. 1117-1128, 1999.

[20] J. Kershaw, B. A. Ardekani, and I. Kanno, "Application of Bayesian inference to fMRI data analysis," IEEE Transactions on Medical Imaging, vol. 18, pp. 1138-53, 1999.

[21] M. Hilton, T. Odgen, D. Hattery, G. Eden, and J. Jawerth, "Wavelet Denoising of Functional MRI Data," in Wavelets in Medicine and Biology, A. Aldroubi and M. Unser, Eds. Boca Raton: CRC Press, 1996, pp. 93-114.

[22] S. Zaroubi and G. Goelman, "Complex denoising of MR data via wavelet analysis: Application for functional MRI," Magnetic Resonance Imaging, vol. 18, pp. 59-68, 2000.

[23] U. E. Ruttimann, M. Unser, R. R. Rawlings, D. Rio, N. F. Ramsey, V. S. Mattay, D. W. Hommer, J. A. Frank, and D. R. Weinberger, "Statistical Analysis of Functional MRI Data in the Wavelet Domain," IEEE Transactions on Medical Imaging, vol. 17, pp. 142 -154, 1998.

[24] S. Mallat and W. L. Hwang, "Singularity detection and processing with wavelets," IEEE Transactions on Information Theory, vol. 38, pp. 617-643, 1992.

[25] M. J. Shensa, "The discrete wavelet transform: Wedding the a trous and Mallat algorithms," IEEE Transactions on Signal Processing, vol. 40, pp. 2464-2482, 1992.

[26] M. Unser, A. Aldroubi, and S. J. Schiff, "Fast implementation of the continuous wavelet transform with integer scales," IEEE Transactions on Signal Processing, vol. 42, pp. 3519-3523, 1994.

[27] I. Koren and A. Laine, "A discrete dyadic wavelet transform for multidimensional feature analysis," in Time Frequency and Wavelets in Biomedical Signal Processing, IEEE press series in Biomedical Engineering, M. Akay, Ed. New York: IEEE Press, 1998, pp. 425-448.

[28] M. Unser, A. Aldroubi, and M. Eden, "On the asymptotic convergence of B-spline wavelets to Gabor functions," IEEE Transactions on Information Theory, vol. 38, pp. 864-872, 1992.

[29] J. Babaud, A. P. Witkin, M. Baudin, and R. O. Duda, "Uniqueness of the Gaussian kernel for scale-space filtering," IEEE Transactions on Pattern Analysis and Machine Intelligence, vol. 8, pp. 26-33, 1986.

[30] I. Daubechies, Ten Lectures on Wavelets. Philadelphia, PA: Siam, 1992.

[31] S. Mallat, $A$ Wavelet Tour of Signal Processing. San Diego, CA: Academic Press, 1998.

[32] C. J. G. Evertsz, K. Berkner, and W. Berghorn, "A Local Multiscale Characterization of Edges Applying the Wavelet Transform," presented at Nato A.S.I, Fractal Image Encoding and Analysis, Trondheim, pp. 1-19, 1995.

[33] T.-C. Hsung, D. P.-K. Lun, and W.-C. Siu, "Denoising by Singularity Detection," IEEE Transactions on Signal Processing, vol. 47, pp. 3139-3144, 1999.

[34] R. D. Nowak, "Wavelet-Based Rician Noise Removal for Magnetic Resonance Imaging," IEEE Transactions on Image Processing, vol. 8, pp. 1408-1419, 1999.

[35] H. Gudbjartsson and S. Patz, "The Rician distribution of noisy MRI data," Magnetic Resonance in Medicine, vol. 34, pp. 910-914, 1995.

[36] R. P. Woods, S. T. Grafton, C. J. Holmes, S. R. Cherry, and J. C. Mazziotta, "Automated image registration: I. General methods and intrasubject, intramodality validation," Journal of Computer Assisted Tomography, vol. 22, pp. 139-52, 1998.

[37] D. L. Donoho, "De-noising by Soft-Thresholding," IEEE Transactions on Information Theory, vol. 41, pp. 613-627, 1995. 